\title{
Effect of different slaughtering methods on metabolites of broiler chickens using Ultra High-Performance Liquid Chromatography-Time of Flight-Mass Spectrometry (UHPLC-TOF-MS)
}

\author{
${ }^{1}$ Ali, N.S.M., ${ }^{1}$ Zabidi, A.R., ${ }^{1}$ Manap, M.N.A., ${ }^{2}$ Zahari, S.M.S.N.S. and ${ }^{1,3, *}$ Yahaya, N. \\ ${ }^{1}$ Department of Food Biotechnology, Faculty of Science and Technology, Universiti Sains Islam Malaysia, \\ 71800, Nilai, Negeri Sembilan, Malaysia \\ ${ }^{2}$ Department of Industrial Chemical Technology, Faculty of Science and Technology, Universiti Sains Islam \\ Malaysia, 71800, Nilai, Negeri Sembilan, Malaysia \\ ${ }^{3}$ International Fatwa and Halal (iFFAH) Center, Universiti Sains Islam Malaysia, 71800, Nilai, Negeri \\ Sembilan, Malaysia
}

\author{
Article history: \\ Received: 31 July 2019 \\ Received in revised form: 25 \\ October 2019 \\ Accepted: 12 December 2019 \\ Available Online: 10 \\ February 2020
}

Keywords:

Broiler chickens,

Halal,

Non-Halal,

Metabolomics,

Metabolites Profile

DOI:

https://doi.org/10.26656/fr.2017.4(S1).S06

\begin{abstract}
Islamic study defined Halal meat as a "thoyyiban" (clean) food source. Halal meat is produced by following slaughtering procedure as determined by the Islamic jurisprudence. Slaughtering methods have gained a worldwide discussion as animal welfare becomes a concern. However, there is lacking of scientific facts to prove which slaughtering methods produce better physiological effects on animals from metabolomics view. Therefore, metabolomics approach by Liquid Chromatography-Time of Flight-Mass Spectrometry (LC-TOF-MS) was used in this study to understand how the metabolites in poultry change when subjected to different slaughtering processes. The broiler chickens were subjected to Halal (Islamic tradition) and non-Halal slaughtering method (neck poking) where pectoral major muscle tissues from the slaughtered meat were selected for UHPLC-TOF-MS analysis. Metabolome data highlighted multiple pathways affected by slaughtering methods including glucose, amino acid, inosine, hypoxanthine and arginine. Higher utilization of energy in non-Halal slaughtering process was observed as indicated by the increase of gluconeogenesis and amino acid breakdown. The result from this study indicated that the method of slaughter affects the metabolites profile of poultry.
\end{abstract}

\section{Introduction}

The definition of food security includes the accessibility to nutritious food (Ayala and Meier, 2017). This encompasses the definition of halal and Thoyyiban according to Islam. In developing halal economic sector, the foods or products must meet the halal and Thoyyiban aspects. This requires procedures that must be followed and monitored along the supply chains beginning from farm to fork (Asa, 2017). In Malaysia, food products that are certified with halal signifying the products are permissible and acceptable due to the whole concept of halal determined by Islamic jurisprudence which also includes hygiene, sanitation and safety aspect. However, the production methods (for instance slaughtering method) and distribution of food products (halal foods distribute with non-halal foods during transportation) create numbers of issues. Disputes on various slaughtering methods spring up when there is a potential of slaughtered meat did not meet the halal requirement and Halal slaughtering method is labeled as inhumane.

Previous research conducted by Mohamed and Mohamed (2012); Wong (2014); Hafiz et al. (2015); and Salwani et al. (2015) discovered different slaughtering methods including halal slaughtering method and nonhalal slaughtering method did affect the physical quality of the chicken meat in term of $\mathrm{pH}$, color, water holding capacity, lipid oxidation, haem iron content, texture and mineral content. However, there are no scientific facts of metabolites profiles on chicken subjected to different slaughtering methods. Therefore, this study is aimed to elucidate the effect of halal and non-halal slaughtering method on chicken metabolite profile using metabolomics fingerprinting.

\section{Materials and methods}

\subsection{Ethics statement}

This study was carried out in strict accordance with 
the recommendations. The procedure to conduct the study was approved by the Animal Ethics Committee of Universiti Sains Islam Malaysia.

\subsection{Research methodology}

The study was carried out at Universiti Sains Islam Malaysia (USIM), Nilai. Two groups of male chickens, (Gallus gallus domesticus) (halal and non-halal slaughtering method), aged 60 days with approximately the same weight $(2 \mathrm{~kg})$ were purchased from a local farm in Kajang. Each group represented by four chickens.

\subsection{Halal slaughtering method}

The chickens were slaughtered according to the Islamic traditions by severing the jugular veins, carotid arteries, trachea and the esophagus. This method was performed without stunning.

\subsection{Non-halal slaughtering method}

Chickens in this group were slaughtered by using a sharp-pointed object to poke the neck of the chicken to create a small hole for blood drainage. After slaughter, chickens were immediately processed.

\subsection{Metabolic extraction}

Metabolite extraction was carried out by using the method described by González-Peña et al. (2017) with modifications. The tissue of pectoralis major muscle was homogenized to a fine powder by rapid freezing in liquid nitrogen. The freeze-dried chicken powder $(250 \mathrm{mg})$ was extracted with $1 \mathrm{~mL}$ cold methanol/water (1:1). The sample was centrifuged for $10 \mathrm{mins}$ at $14000 \mathrm{rpm}$ at room temperature. Supernatants was collected in $1.5 \mathrm{~mL}$ centrifuge tubes (Eppendorf) and stored in $-80^{\circ} \mathrm{C}$ until further analysis.

\subsubsection{Liquid Chromatography-Time of Flight-Mass Spectrometry analyses}

UHPLC was performed on ACQUITY UPLC IClass system from Waters (Manchester, UK), consisting of a binary pump, a vacuum degasser, an autosampler and a column oven. Compounds were chromatographically separated using a column ACQUITY UPLC HSS T3 (100 mm x $2.1 \mathrm{~mm}$ x $1.8 \mu \mathrm{m})$ also from Waters, maintained at $40^{\circ} \mathrm{C}$. A linear binary gradient of water $(0.1 \%$ formic acid $)$ and acetonitrile (mobile phase B) was used as mobile phase A and B respectively. The mobile phase composition was changed during the run as follows: $0 \mathrm{~min}, 1 \% \mathrm{~B} ; 0.5$ mins, $1 \% \mathrm{~B}$; 16.00 mins, $35 \% \mathrm{~B}$; 18.00 mins, $100 \% \mathrm{~B}$; $20.00 \mathrm{mins}, 1 \% \mathrm{~B}$. The flow rate was set to $0.6 \mathrm{~mL} / \mathrm{min}$ and the injection volume was $1 \mu \mathrm{L}$. The UHPLC system was coupled to a Vion IMS QTOF hybrid mass spectrometer from Waters (Manchester, UK), equipped with a Lock Spray ion source. The ion source was operated in negative electrospray ionization (ESI) mode under the following specific conditions: capillary voltage, $1.50 \mathrm{kV}$; reference capillary voltage, $3.00 \mathrm{kV}$; source temperature, $120^{\circ} \mathrm{C}$; desolvation gas temperature, $550^{\circ} \mathrm{C}$; desolvation gas flow, $800 \mathrm{~L} / \mathrm{hr}$, and cone gas flow, $50 \mathrm{~L} / \mathrm{hr}$. Nitrogen $(>99.5 \%)$ was employed as desolvation and cone gas. Data were acquired in highdefinition $\mathrm{MS}^{\mathrm{E}}\left(\mathrm{HDMS}^{\mathrm{E}}\right)$ mode in the range $\mathrm{m} / \mathrm{z} 50$ 1500 at $0.1 \mathrm{~s} / \mathrm{scan}$. Thus, two independent scans with different collision energies (CE) were alternatively acquired during the run: a low-energy (LE) scan at a fixed $\mathrm{CE}$ of $4 \mathrm{eV}$, and a high- energy (HE) scan where the $\mathrm{CE}$ was ramped from 10 to $40 \mathrm{eV}$. Argon (99.999\%) was used as collision-induced-dissociation (CID) gas.

\section{Results and discussion}

\subsection{Carbohydrate metabolism}

LC-TOF-MS system coupled with an ESI was used to obtain metabolomics data of halal and non-halal chicken meat. The preliminary data was displayed in LCTOF-MS spectra (BPI Counts Vs Retention time, Figures 1 and 2). Metabolites detected in halal chicken and non-halal chicken and in both halal and non-halal chicken were illustrated in diagram (Figure 3). LC-TOFMS spectra showed the presence of isomaltose in nonhalal chicken meat (Figure 2), but no detection of isomaltose in halal chicken meat (Figure 1). This indicated that gluconeogenesis has been elevated in nonhalal chicken meat since isomaltose is a byproduct of glucose metabolism.

It was assumed that non-halal chicken was under stress before the slaughtering process. Under stress condition, the body will respond by increasing the production and release of nutrients (Jasterbski et al., 2017). Glucose acts as the major energy source for most tissues notably the brain, thus it is considered as one of the essential nutrients (Svihus, 2014). The glucose can be supply systemically by two metabolic pathways, either by the breakdown of stored glycogen or through gluconeogenesis (Jasterbski et al., 2017).

Digestion of starch in poultry is the function of pancreatic amylase which is produced endogenously in the animal (Sanan, 2017). Figure 4 shows starch and sucrose metabolism pathway in chicken. The amylase will break the starch into shorter polymers called dextrin which can be further hydrolyzed into units of maltose, isomaltose and glucose (Donald et al., 2002). The maltose and isomaltose can be hydrolyzed from intestinal secretions producing the enzymes maltase and isomaltase that will further hydrolyze the carbohydrate 


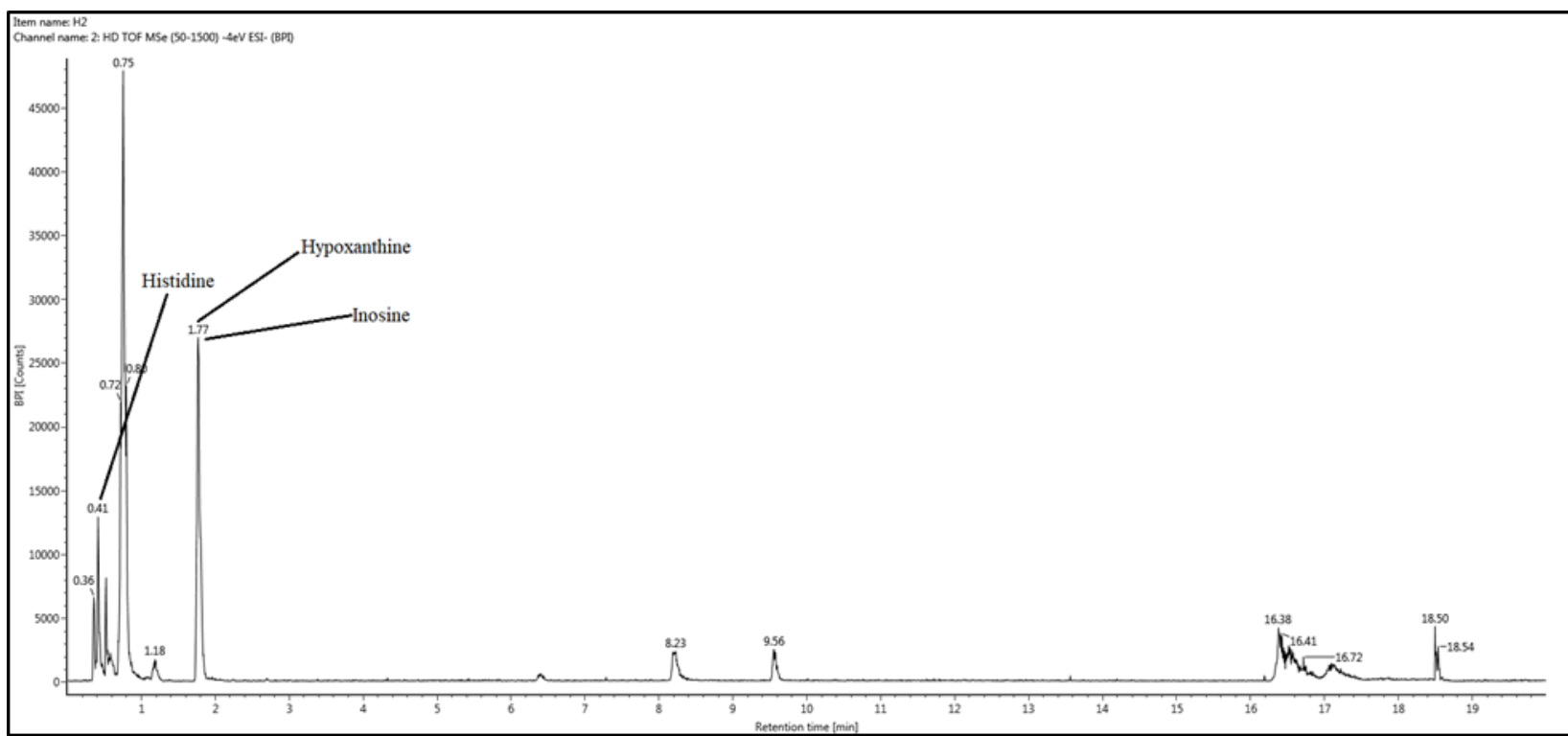

Figure 1. UHPLC-TOF spectra of Halal chicken pectoralis major muscle tissue.

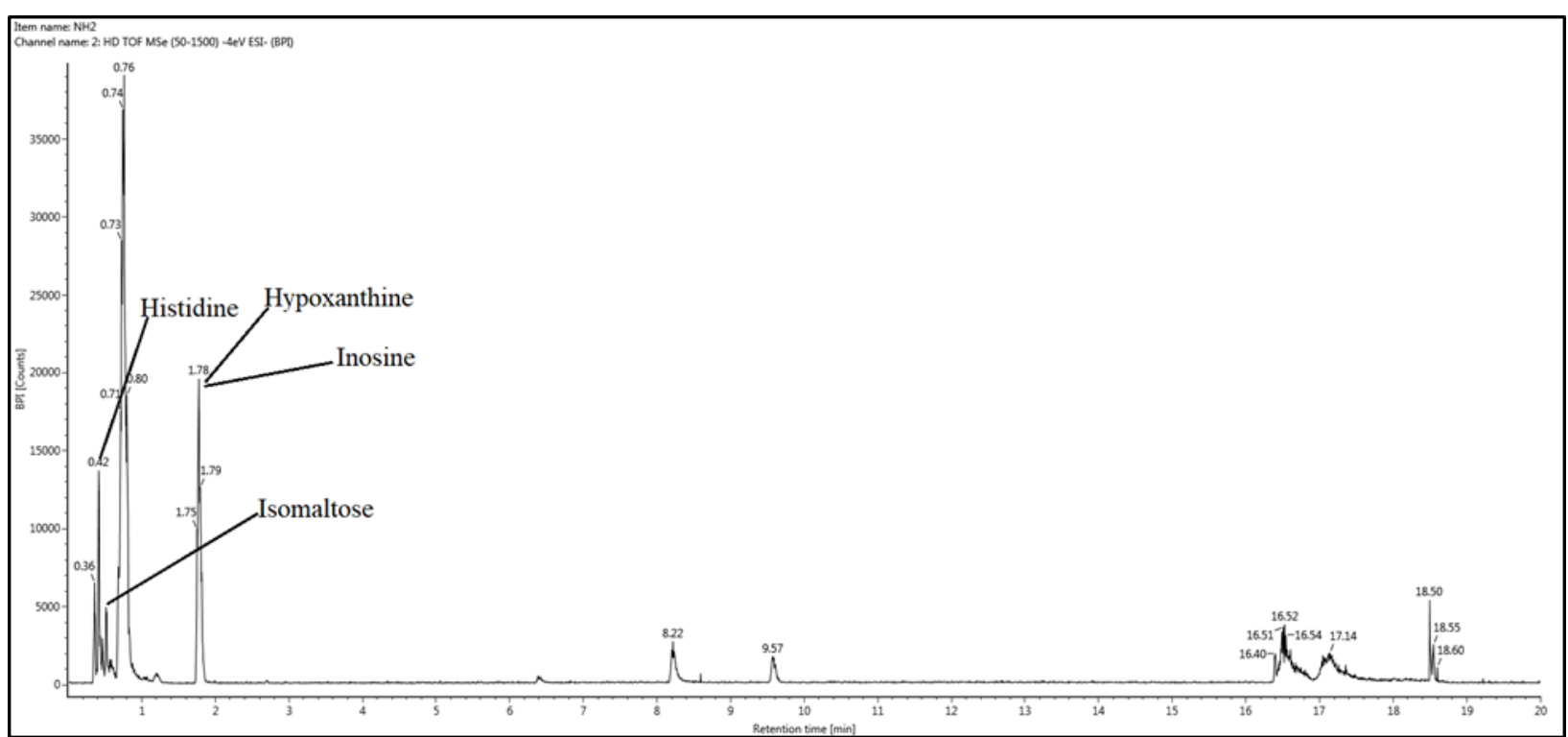

Figure 2. UHPLC-TOF spectra of non-Halal chicken pectoralis major muscle tissue

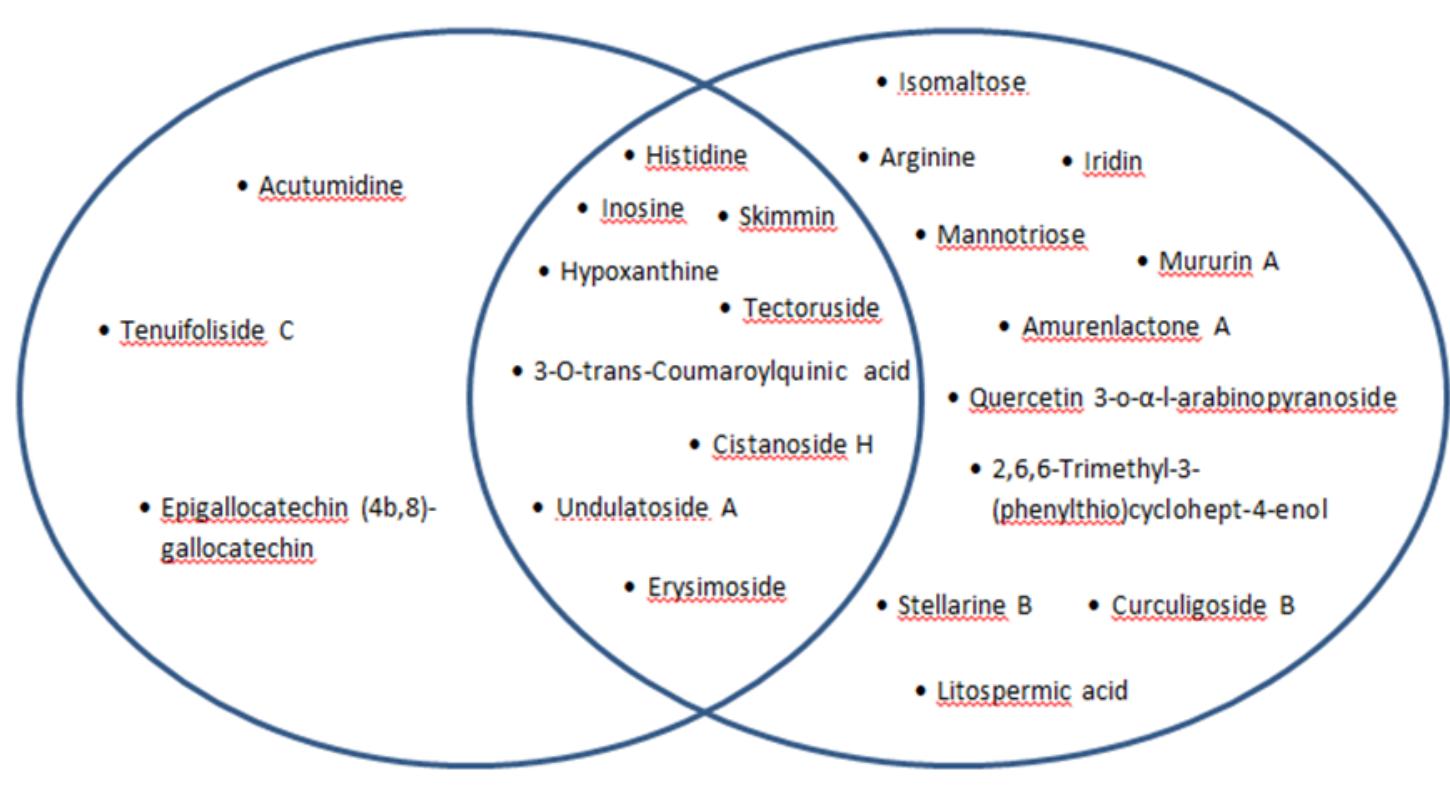

(a)

(b)

Figure 3. (a) Metabolites that are detected in halal chicken meat. (b) Metabolites that are detected in non-halal chicken meat. (c) Metabolites that are detected in both halal and non-halal chicken meat. 


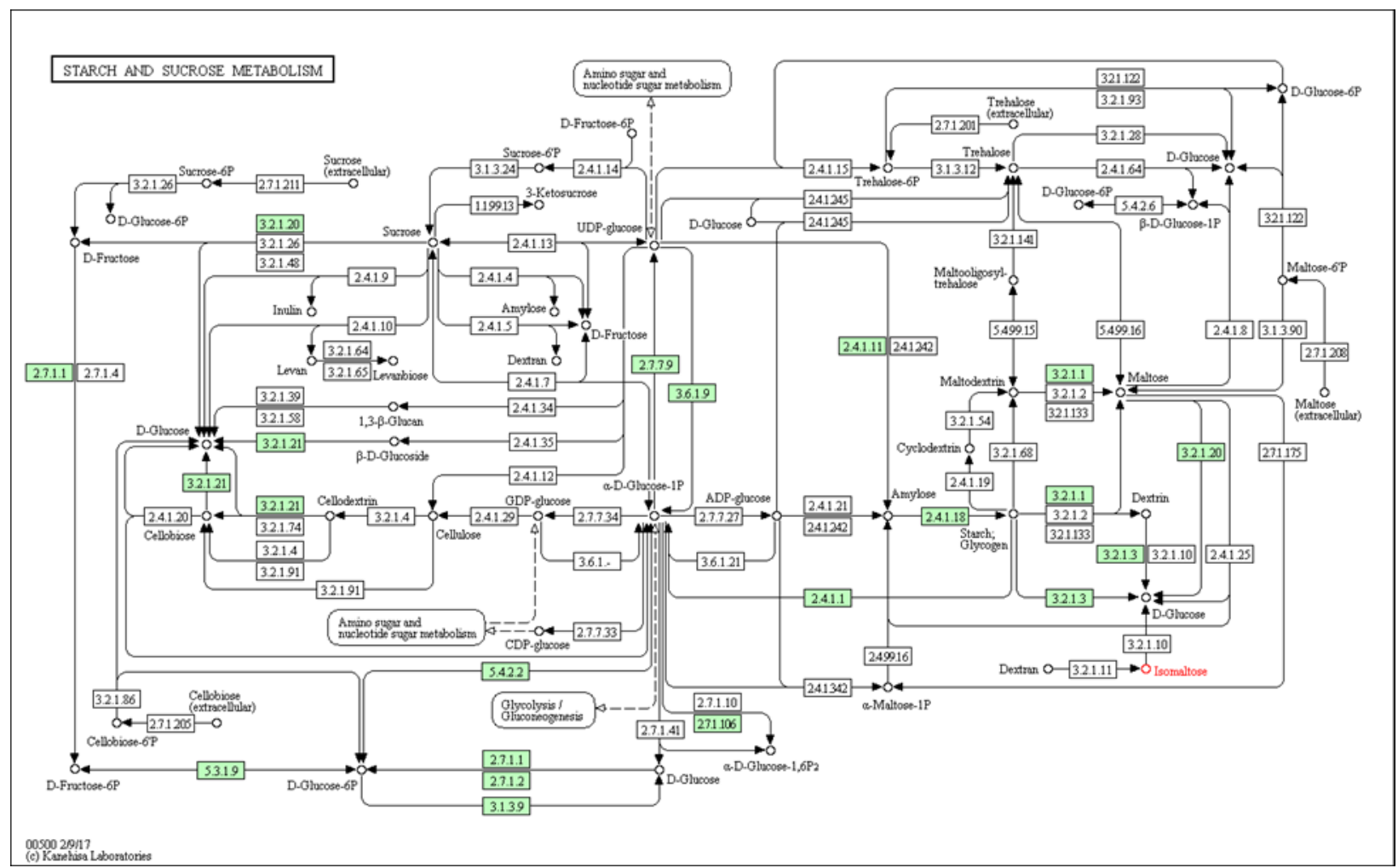

Figure 4. Starch and sucrose metabolism pathway in chicken (Gallus gallus domesticus)

into glucose units. The glucose then, can be actively absorbed in the intestine of poultry. Carbohydrate that is being absorbed can be metabolized: 1) for lipid formation, 2) for structural components formation such as chondroitin sulfate in cartilage, 3 ) for production of chemical energy that can be utilized by the poultry for productive purposes, and 4) during acute stressful situation where synthesizing of reserve glucose source called glycogen will occur which then will restore the depleted energy (Donald et al., 2002). Therefore, this study suggested that the increase of glucose export in non-halal chicken is due to higher energy utilization during the struggling of death and stress faced by the chicken.

\subsection{Amino acid metabolism}

LC-TOF-MS spectra showed a lower concentration of histidine in non-halal chicken meat (Figure 2) as compared to halal chicken meat (Figure 1). Histidine is categorized as basic amino acids including lysine and arginine. In poultry, these amino acids are considered essential as the body cannot produce these compounds endogenously (Jun et al., 2018). These amino acids exert their roles in several metabolite pathways. Metabolite roles of arginine are showed through the byproduct production including nitric oxide (NO), L-ornithine (LOrn), polyamines, proline, glutamate, creatine and agmantine (Morris, 2004). Histidine is transformed to histamine by enzyme histidine decarboxylase and also histidine degraded to glutamate (Stifel and Herman, 1971).
Amino acid also plays its role as an energy source in poultry apart from functioning as substrates for proteins biosynthesis and physiological messengers in the body (Yamane et al., 2019). Amino acid can be grouped into ketogenic amino acid and glucogenic amino acids depending on byproducts produced after hydrolyzation. Amino acid that hydrolyzed to acetyl-CoA or acetoacetyl -CoA fall into the ketogenic amino acid group due to its ability to give rise to ketone bodies or fatty acid. Amino acids that degraded to pyruvate, $\alpha$-ketoglutarate, succinyl -CoA, fumarate or oxaloacetate are termed as glucogenic amino acids (Yamane et al., 2019). Both hydrolyzation processes contribute to energy production in poultry.

Due to increased metabolic demands during nonhalal slaughtering, the chicken is likely to increase the breakdown of amino acids for energy as shown by the metabolome data. Therefore, it is hypothesized that the lower concentration histidine which is $13500 \mathrm{BPI}$ in nonhalal chicken meats may be due to increased recruitment and utilization of this amino acid in order to provide energy substrates for the cell at the brink of death.

\subsection{Inosine metabolism}

It was observed that different slaughtering methods had affected the inosine metabolism of chicken. Figure 2 shows that a higher concentration of inosine was detected in non-halal chicken meat compared to halal chicken meat (Figure 1). The lower concentration of hypoxanthine which is 20000 BPI was observed in nonhalal chicken meat as recorded in the metabolome data (Figure 2). Inosine represents a metabolite of ATP 
degradation which can be converted to hypoxanthine released into the blood circulation (McConell et al., 2005; Bishop, 2010). A higher concentration of hypoxanthine (27000 BPI) was recorded in halal chicken suggested that muscle ATP could have been catabolized to supply energy within the cell. The increase of energy depletion was observed due to the lower value of inosine in halal breast muscle and the increasing concentration of hypoxantine as recorded by the metabolome data.

However, it is interesting to note how inosine and its byproducts affect the flavor profile quality of poultry. Amino acids and inosine monophosphate (IMP) are among the major compounds contributing to meat flavor (Zhu and Lu, 1996). IMP is considered as a key index of meat flavor as it plays a major role in meat flavor (Suzuki et al., 1994; Fuiymura and Chen, 1998). Hall (1964) stated that IMP and its corresponding nucleoside and base, inosine and hypoxanthine are normally found in meat and these substances are derived from the degradation of adenosine triphosphate (ATP). Therefore, the degradation of ATP is a desirable metabolic activity, as it can contribute to flavor quality of meat.

\section{Conclusion}

Metabolome data illustrated the metabolomics activity of gluconeogenesis, amino acid breakdown, inosine hydrolyzation as well as hypoxanthine production in broiler chickens when subjected to different slaughtering methods. Non-halal slaughter resulted in higher utilization of energy as indicated by the elevated amino acid and glucose breakdown. Halal slaughtering method favors less energy usage, hence keeping the welfare of the animal as well as the meat quality.

\section{Acknowledgement}

This research is supported by the Malaysian Ministry of Higher Education, FRGS/1/2017/WAB01/USIM/02/1.

\section{References}

Asa, R.S. (2017). Malaysian Halal Certificate: It's Religious Significance and Economic Value. Shariah Journal, 25(1), 137-156.

Ayala, A. and Meier, B.M. (2017). A Human Rights Approach to The Health Implications of Food and Nutrition Insecurity. Public Health Reviews, 38(10). https://doi.org/10.1186/s40985-017-0056-5

Bishop, D. (2010). Dietary Supplements and Team-Sport Performance. Sports Medicine, 40(12), 995-1017. https://doi.org/10.2165/11536870-000000000-00000

Donald, D.B., William, D. and Weaver. (2002).
Commercial Chicken Meat and Egg Production. $5^{\text {th }}$ ed. United States: Kluwer Academic Publishers.

Fuiymura, W.L. and Chen. L.J. (1988). Change of Plasma Testosterone in Male Silkies. Journal of Chinese Animal, 5, 24-25.

González-Peña, D., Dudzik, D; García, A., de Ancos, B., Barbas, C. and Sánchez-Moreno, C. (2017). Metabolomic Fingerprinting in The Comprehensive Study of Liver Changes Associated with Onion Supplementation in Hypercholesterolemic Wistar Rats. International Journal of Molecular Sciences, 18(2), 1-16. https://doi.org/10.3390/ijms18020267

Hafiz, A., Hassan, Z. and Manap, M.N. A. (2015). Effect of Slaughtering Methods on Meat Quality Indicators, Chemical Changes and Microbiological Quality of Broiler Chicken Meat during Refrigerated Storage. IOSR Journal of Agriculture and Veterinary Science, 8(9), 2319-2372.

Hall, K.H. (1964). Sensory and Chemical Comparisons of Certain Flavor Constituents of Chicken and Turkey Meat. Pullman: Washington State University, Pullman, PhD. Dissertation.

Jun, Y., Haiming, Y., Zhiyue, W., Hang, D., Lei, X. and Chuang, L. (2017). Effects of Arginine on the Growth Performance, Hormones, Digestive Organ Development and Intestinal Morphology in the Early Growth Stage of Layer Chickens. Italian Journal of Animal Science, 17(4), 1077-1082. https:// doi.org/10.1080/1828051X.2018.1434692

McConell, G.K., Shinewell, J., Stephens, T.J., Stathis, C.G., Canny, B.J., and Snow, R.J. (2005). Creatine Supplementation Reduces Muscle Inosine Monophosphate during Endurance Exercise in Humans. Medicine and Science in Sports and Exercise, 37(12), 2054-2061. https:// doi.org/10.1249/01.mss.0000179096.03129.a4

Mohamed, B and Mohamed, I. (2012). The Effects of Residual Blood of Carcasses on Poultry Technological Quality. Food and Nutrition Sciences, 3, 1382-1386. https://doi.org/10.4236/ fns.2012.310181

Morris, S.M.Jr. (2004). Enzymes of Arginine Metabolism. The Journal of Nutrition, 134(10), 2743 -2747. https://doi.org/10.1093/jn/134.10.2743S

Salwani, M.S., Adeyemi, K.D., Sarah, S.A., Vejayan, J., Zulkifli, I. and Sazili, A.Q. (2015). Skeletal Muscle Proteome and Meat Quality of Broiler Chickens Subjected to Gas Stunning Prior Slaughter or Slaughtered Without Stunning. CyTA - Journal of Food, 14(3), 1-7. https:// doi.org/10.1080/19476337.2015.1112838

Talibov, S. (2017). Starch Digestion in Broiler Chickens: 
A Literature Study and an In vitro Comparison with Pigs. Poland: Poznań University of Life Sciences, MSc. Dissertation.

Jasterbski, S.F., Lamont, S.J. and Schmidt, C.J. (2017). Chicken Hepatic Response to Chronic Heat Stress using Integrated Transcriptome and Metabolome Analysis. PLoS One, 12(7), e0181900. https:// doi.org/10.1371/journal.pone.0181900

Stifel, F.B. and Herman, R.H. (1971). Histidine metabolism. The American Journal of Clinical Nutrition 24(2), 207-217. https://doi.org/10.1093/ ajen/24.2.207

Suzuki, A.N., Homma, A. and Fukuda Y.T. (1994). Effect of High Pressure Treatment on the FlavorRelated Components in Meat. Journal of Meat Science, 37(3), 369-379. https:// doi.org/10.1016/0309-1740(94)90053-1

Svihus, B. (2014). Starch Digestion Capacity of Poultry. Poultry Science, 93(9), 2394-2399. https:// doi.org/10.3382/ps.2014-03905

Wong, M.M. (2014). The Effect of Non-Stunned and Stunned Halal Slaughter Method on Broiler Breast Meat Quality. MOJ Food Processing and Technology, 1(3), 1-10. https://doi.org/10.15406/ mojfpt.2015.01.00014

Yamane, H., Kurauchi, I., Denbowl, D.M. and Furuse, M. (2009). Central Functions of Amino Acids for the Stress Response in Chicks. Asian-Australasian Journal of Animal Science, 22(2), 296 - 304. https:// doi.org/10.5713/ajas.2009.r.02

Zhu, G.B. and Lu, H.J. (1996). Foodstuff Flavor Principium and Technique. Beijing: Beijing University Press. 